\title{
21 Anlagen
}

\subsection{Gedicht „Glück“ von Friedrich Halm (1806-1871)}

„Was jeder sucht und was so wen'ge kennen, Wonach wir alle jagen stets und rennen, Wofür selbst Greise glühen noch und brennen, Glück, was ist Glück? Wer weiß es mir zu nennen?

Befriedigung? - Das Herz kennt keinen Frieden! Und Ruhe? Wem war jemals sie beschieden? Freiheit vielleicht? - Doch wer ist frei hienieden? Glück, was ist Glück? Wer hat es je entschieden?

Dem ist es Reichtum, jener nennt es Macht; Dort grünt es einem in des Lorbeers Pracht, Der findet es in wüst durchschwelgter Nacht, Und dieser, wenn es sie beim Buch durchwacht! Glück ist, was jeder sich als Glück gedacht!

Und träte einer nun zu mir heran, Und spräche flehend: Zeige mir die Bahn Zum Glück, zum Glück, nach dem wir alle jagen, Die Worte müßt' ich ihm zur Antwort sagen:

Erst liebe, was auch deine Neigung wähle, Ein Weib, ein Kind, Kunst, Wissenschaft, Natur, Doch lieb' es ganz aus voller trunkner Seele, Und leb' und web' in diesem einen nur! Laß ganz aus dir des Ich's Bewußtsein schwinden, Tauch' unter wie ins Meer in dein Empfinden, Beglückend nur fühl' selber dich beglückt, Gib ganz dich auf, und lerne froh entzückt, Je mehr du gabst, nur reicher stets dich finden.

Dann schaffe, was es sei, nach deinen Gaben, Ein Lied, ein Bild; treib Handel, führ' den Pflug: Doch mußt du hoch das Ziel gesteckt dir haben, Und was du leistest, sei dir nie genug! Laß nie die Kraft, den Willen dir erschlaffen, Vom bessern dich zum besten aufzuraffen; Nur wenn dein Geist nach Fortschritt ewig geizt, Wenn ewig ihn Vollendung lockt und reizt, Dann lebst du erst; es leben nur, die schaffen!

Und dann - dann stirb, denn besser nie erfahren Der Liebe Glück, des Schaffens Drang und Lust, 
Als sie verglimmen fühlen in der Brust,

Und traurig überleben, was wir waren.“1

\title{
21.2 Robert Ley: „Kraft durch Freude“ (1937)
}

\author{
„,Kraft durch Freude“ ist Arbeit an der Gestaltung \\ des gesamten sozialen Lebens. Denn ,Kraft durch \\ Freude، ist ja nicht allein Gestaltung der Freizeit - \\ ,Kraft durch Freude‘ bedeutet wahre Gemeinschaft, \\ bedeutet die neue Gesellschaft des nationalen Staates, \\ bedeutet die Neuformung des gesamten deutschen \\ Lebens überhaupt! \\ Weder Gewaltmaßnahmen noch Gesetze können \\ Das Glück der Menschen begründen, sondern nur \\ solche Organisationen, die der seelischen und völk- \\ ischen Haltung eines Volkes entsprechen und da- \\ durch seine lebensbejahenden Kräfte zur Wirkung \\ bringen. ,Kraft durch Freude“ ist die große Gemein- \\ schaft, in der die aus dem tiefsten Wesen des deut- \\ schen Volkes kommende Lebenskraft und Lebens- \\ freude wirk und lebt. \\ Mit stolzer Freude schaffen wir weiterhin an \\ diesem Werk, das den deutschen Menschen in das \\ Reich der Schönheit und der Kraft führt, und das in \\ seinem unbändigen Glauben an die Lebensfreude ein \\ mächtiger Faktor ist zum Frieden der Völker! \\ Vorwärts mit Adolf Hitler!“²
}

1 Gedicht des Dichters Friedrich Halm (1806-1871), eigentlich Eligius Franz Joseph Freiherr von Münch-Bellinghausen, österreichischer Dramatiker, Lyriker, Novellist und Generalintendant des Wiener Hoftheaters. In gekürzter Form wurde dieses Gedicht in Gerlings Ratgeber „Die Gymnastik des Willens. Praktische Anleitungen zur Erhöhung der Energie und Selbstbeherrschung, Kräftigung von Gedächtnis und Arbeitslust durch Stärkung der Willenskraft ohne fremde Hilfe“ im Kapitel „Die Jagd nach dem Glück“ abgedruckt. Vgl. Gerling 1920, 170. Originalfassung, Halm 1904. 2 Ley 1937b, „Reichsleiter“ der NSDAP und der „Deutschen Arbeitsfront“ (DAF). 


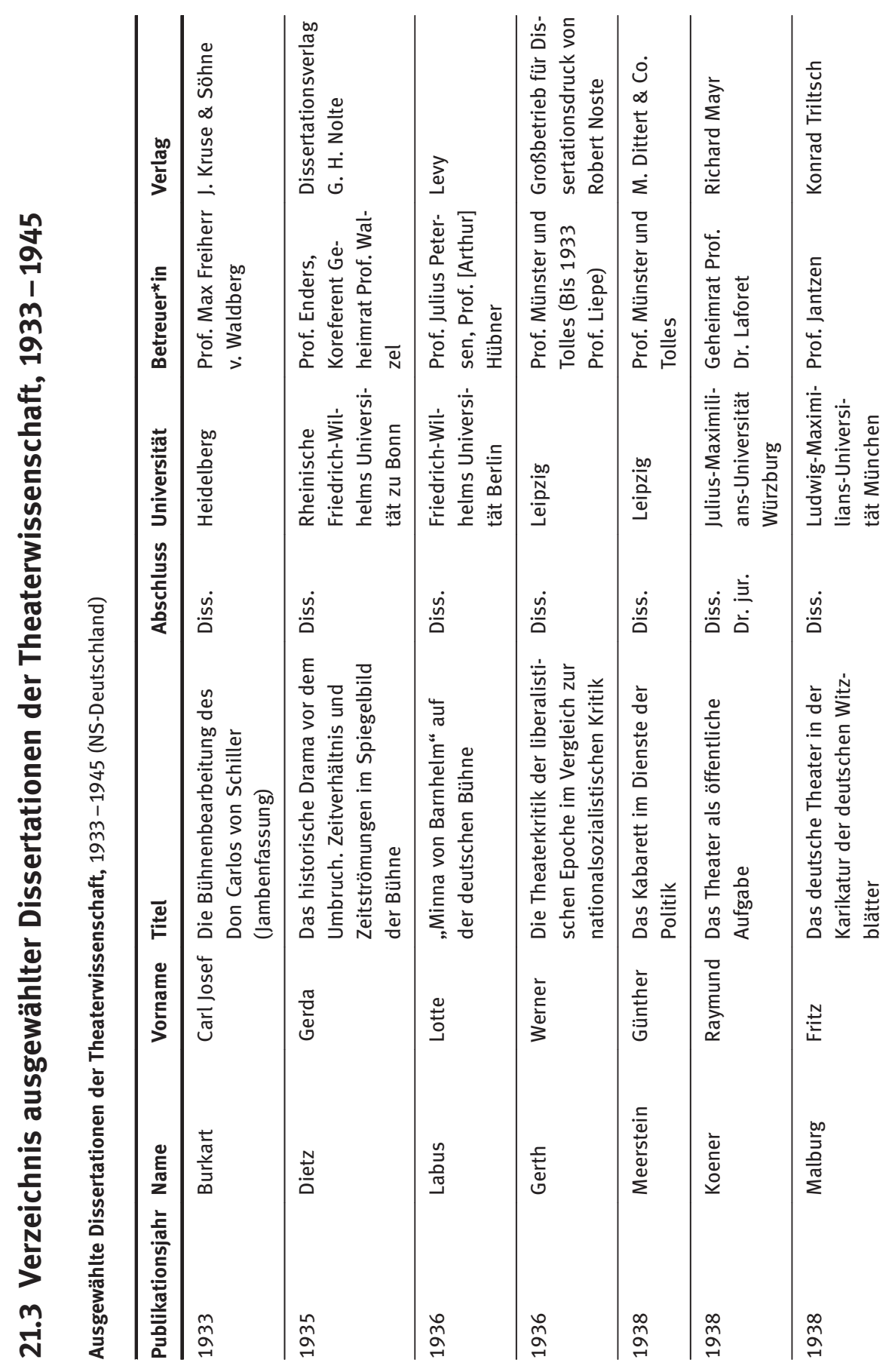




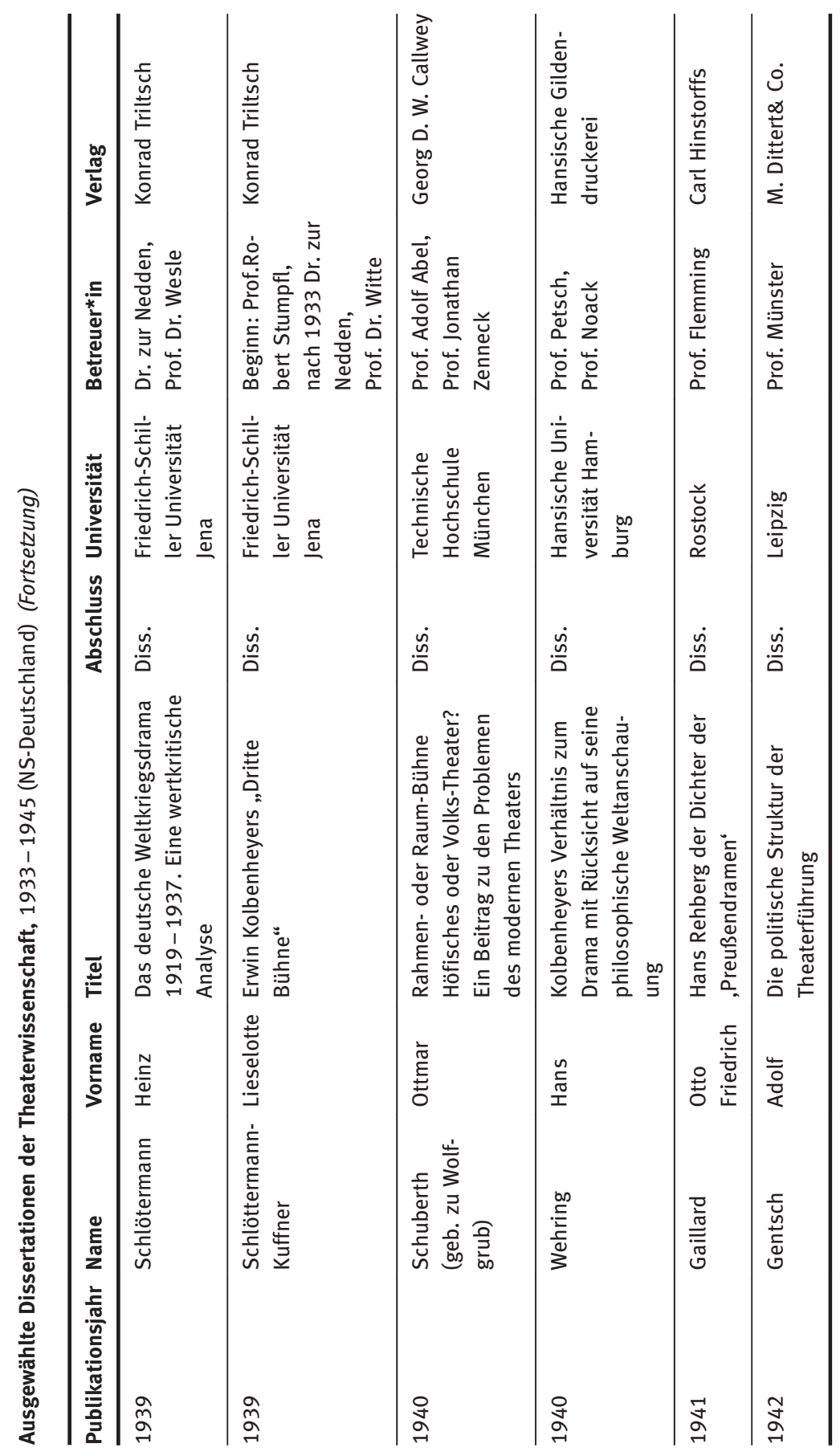




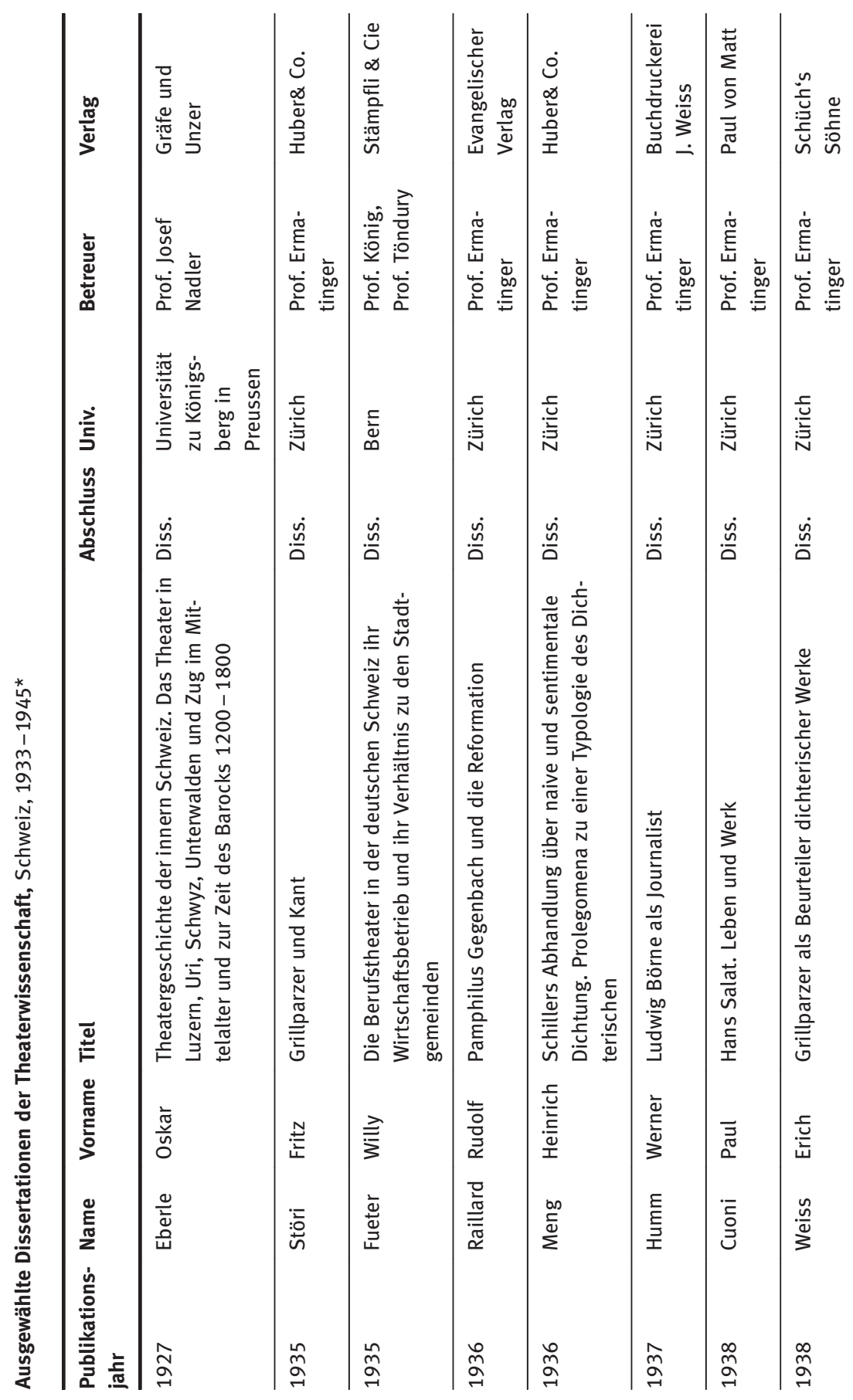




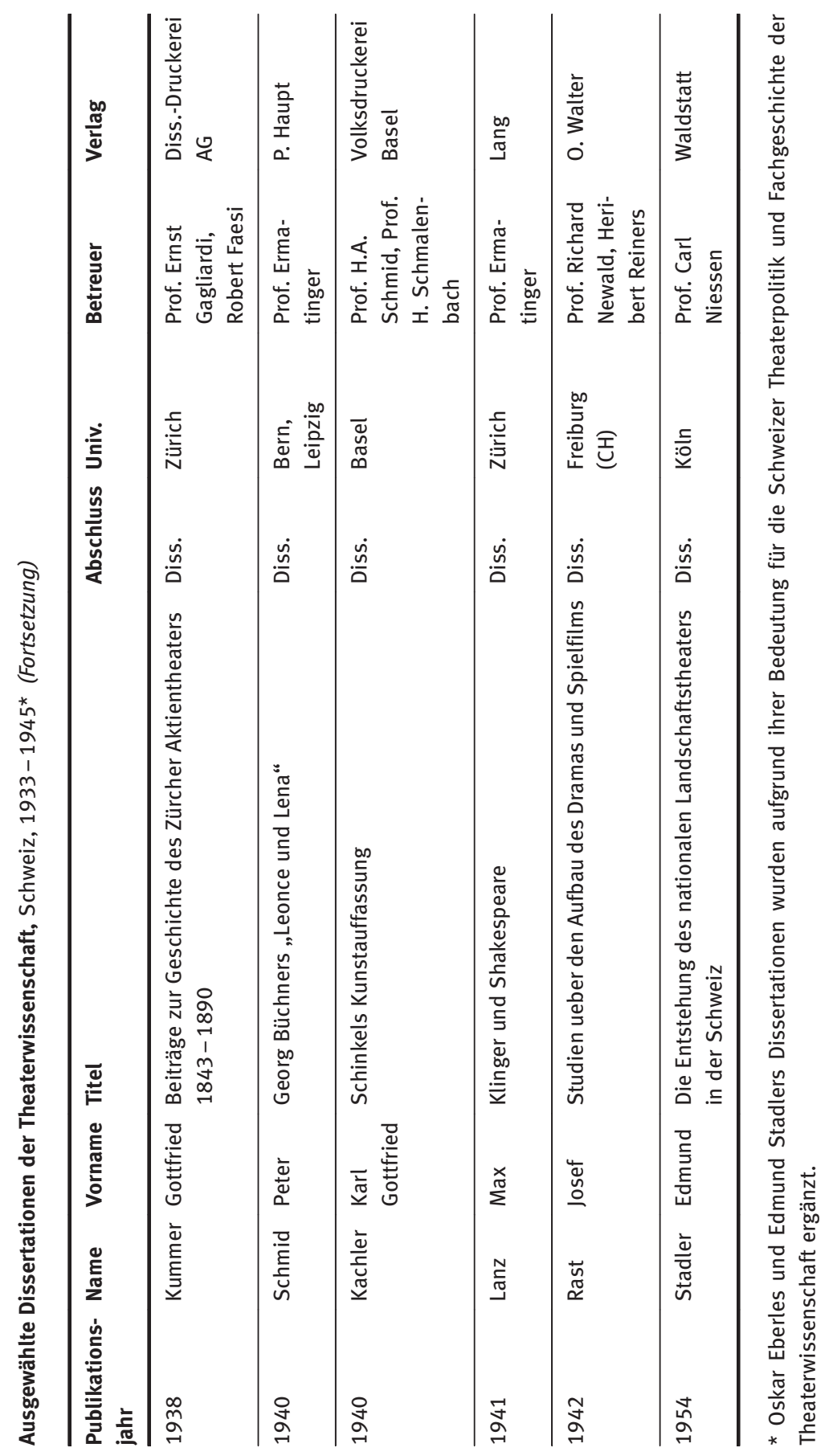




\subsection{Verzeichnis theaterwissenschaftlicher Vorlesungen der Universität Zürich, 1933-1945}

Theaterwissenschaftliche Vorlesungen der Universität Zürich, 1933-1945³

\begin{tabular}{|c|c|c|c|c|c|}
\hline Semester & Nr. & Titel der Veranstaltung & Dozent & Fachbereich & S. \\
\hline \multirow[t]{4}{*}{$\begin{array}{l}\text { WS } \\
1932 / 33\end{array}$} & 330. & Goethe & Prof. Faesi & $\begin{array}{l}\text { Germanische Sprachen } \\
\text { und Literaturen }\end{array}$ & 21 \\
\hline & 331. & $\begin{array}{l}\text { Dramatiker des 19. Jahr- } \\
\text { hunderts }\end{array}$ & P.-D. Muschg & Ebd. & 21 \\
\hline & 336. & Analysen von Dramen & Ders. & Deutsches Proseminar & 21 \\
\hline & 338. & $\begin{array}{l}\text { Theater und Dramen von } \\
\text { Lessing bis in die Gegen- } \\
\text { wart }\end{array}$ & Prof. Faesi & Deutsches Seminar & 21 \\
\hline \multirow[t]{2}{*}{ SS 1933} & 276. & $\begin{array}{l}\text { Ethik: Was ist Glückselig- } \\
\text { keit }\end{array}$ & $\begin{array}{l}\text { P.D. Prof. Eleuthe- } \\
\text { ropolus }\end{array}$ & $\begin{array}{l}\text { Philosophie, Psycholo- } \\
\text { gie, Pädagogik }\end{array}$ & 17 \\
\hline & 353. & $\begin{array}{l}\text { Das schweizerische Dra- } \\
\text { ma der Reformationszeit }\end{array}$ & Prof. Ermatinger & Deutsches Seminar & 21 \\
\hline \multirow[t]{4}{*}{$\begin{array}{l}\text { WS } \\
1933 / 34\end{array}$} & 325. & $\begin{array}{l}\text { Lessing, Herder und der } \\
\text { Sturm und Drang }\end{array}$ & Ders. & $\begin{array}{l}\text { Germanische Sprachen } \\
\text { und Literaturen }\end{array}$ & 20 \\
\hline & 326. & $\begin{array}{l}\text { Schillers Leben und } \\
\text { Werke }\end{array}$ & Ders. & Ebd. & 20 \\
\hline & 327. & Goethes Faust & Ders. & Ebd. & 20 \\
\hline & 333. & $\begin{array}{l}\text { Einführung in die Litera- } \\
\text { turwissenschaft }\end{array}$ & Ders. & Ebd. & 20 \\
\hline \multirow[t]{4}{*}{ SS 1934} & 284. & Was ist Glückseligkeit & $\begin{array}{l}\text { Prof. Eleutheropu- } \\
\text { los }\end{array}$ & $\begin{array}{l}\text { Philosophie, Psycholo- } \\
\text { gie, Pädagogik }\end{array}$ & 18 \\
\hline & 346. & $\begin{array}{l}\text { G. Hauptmann u. andere } \\
\text { mod. Dramatiker }\end{array}$ & Prof. Faesi & $\begin{array}{l}\text { Germanische Sprachen } \\
\text { und Literaturen }\end{array}$ & 21 \\
\hline & 351. & Analyse von Dramen & Prof. Ermatinger & Deutsches Proseminar & 21 \\
\hline & 353. & Schillers Dramen & Ders. & Deutsches Seminar & 22 \\
\hline \multirow[t]{2}{*}{$\begin{array}{l}\text { WS } \\
1934 / 35\end{array}$} & 326. & Goethe & Ders. & $\begin{array}{l}\text { Germanische Sprachen } \\
\text { und Literaturen }\end{array}$ & 19 \\
\hline & 327. & $\begin{array}{l}\text { Dramatiker des 19. Jahr- } \\
\text { hunderts }\end{array}$ & Ders. & Ebd. & 19 \\
\hline
\end{tabular}

3 Verzeichnis der Vorlesungen. Universität Zürich. Zürich: Universität, Archiv, UZH. 
Theaterwissenschaftliche Vorlesungen der Universität Zürich, 1933-1945 (Fortsetzung)

\begin{tabular}{|c|c|c|c|c|c|}
\hline Semester & Nr. & Titel der Veranstaltung & Dozent & Fachbereich & S. \\
\hline & 330. & Hebbel, Ibsen & Prof. Faesi & Ebd. & 19 \\
\hline \multirow[t]{2}{*}{ SS 1935} & 341. & $\begin{array}{l}\text { Der Weltkrieg u. die } \\
\text { deutsche Literatur }\end{array}$ & P.-D. E. Staiger & Ebd. & 20 \\
\hline & 342. & $\begin{array}{l}\text { Übungen an Dramen zur } \\
\text { ästhetischen Urteilsbil- } \\
\text { dung }\end{array}$ & Prof. Faesi & Ebd. & 20 \\
\hline \multirow[t]{7}{*}{$\begin{array}{l}\text { WS } \\
1935 / 36\end{array}$} & 334 & $\begin{array}{l}\text { *Dichtung und Geistes- } \\
\text { leben der deutschen } \\
\text { Schweiz }\end{array}$ & Prof. Ermatinger & Ebd. & 19 \\
\hline & 336. & $\begin{array}{l}\text { Das Problem des Tragi- } \\
\text { schen von Gryphius bis } \\
\text { zur Gegenwart }\end{array}$ & P.-D. E. Staiger & Ebd. & 20 \\
\hline & 337. & $\begin{array}{l}\text { Lessing, Herder und der } \\
\text { Sturm und Drang }\end{array}$ & Prof. Ermatinger & Ebd. & 20 \\
\hline & 338. & $\begin{array}{l}\text { Schillers Leben und } \\
\text { Werke }\end{array}$ & Ders. & Ebd. & 20 \\
\hline & 344. & Analysen von Dramen & Ders. & Deutsches Proseminar & 20 \\
\hline & 346. & $\begin{array}{l}\text { Das Drama des 19. Jahr- } \\
\text { hunderts }\end{array}$ & Ders. & Deutsches Seminar & 20 \\
\hline & 347. & $\begin{array}{l}\text { Theaterwissenschaftliche } \\
\text { Übungen }\end{array}$ & Prof. Faesi & Deutsches Seminar & 20 \\
\hline SS 1936 & - & - & - & - & - \\
\hline \multirow[t]{2}{*}{$\begin{array}{l}\text { WS } \\
1936 / 37\end{array}$} & 343. & $\begin{array}{l}\text { Kolloquium über den } \\
\text { Briefwechsel zwischen } \\
\text { Schiller und Goethe }\end{array}$ & P.-D. E. Staiger & $\begin{array}{l}\text { Germanische Sprachen } \\
\text { und Literaturen }\end{array}$ & 18 \\
\hline & 345. & $\begin{array}{l}\text { Dramatiker des 19. Jahr- } \\
\text { hunderts }\end{array}$ & Prof. Ermatinger & Ebd. & 18 \\
\hline \multirow[t]{4}{*}{ SS 1937} & 376. & $\begin{array}{l}\text { Dramatiker: Hebbel, } \\
\text { Ibsen, Hauptmann }\end{array}$ & Prof. Faesi & Ebd. & 19 \\
\hline & 381. & Analyse von Dramen & Prof. Ermatinger & Deutsches Proseminar & 19 \\
\hline & 383. & $\begin{array}{l}\text { Übungen über Hebbels } \\
\text { Dramen }\end{array}$ & Ebd. & Deutsches Seminar & 19 \\
\hline & 384. & $\begin{array}{l}\text { Österreichische Dichtung } \\
\text { seit Grillparzer }\end{array}$ & Prof. Faesi & Ebd. & 19 \\
\hline
\end{tabular}


Theaterwissenschaftliche Vorlesungen der Universität Zürich, 1933-1945 (Fortsetzung)

\begin{tabular}{|c|c|c|c|c|c|}
\hline Semester & Nr. & Titel der Veranstaltung & Dozent & Fachbereich & S. \\
\hline \multirow[t]{2}{*}{$\begin{array}{l}\text { WS } \\
1937 / 38\end{array}$} & 379. & Dramatiker des 19. Jhdts. & Prof. Ermatinger & $\begin{array}{l}\text { Germanische Sprachen } \\
\text { und Literaturen }\end{array}$ & 21 \\
\hline & 389. & $\begin{array}{l}\text { Das Theater der letzten } \\
\text { hundert Jahre }\end{array}$ & Prof. Faesi & Ebd. & 22 \\
\hline SS 1939 & 397. & Das deutsche Lustspiel & P.-D. E. Staiger & Ebd. & 22 \\
\hline \multirow[t]{2}{*}{$\begin{array}{l}\text { WS } \\
1939 / 40\end{array}$} & 385. & $\begin{array}{l}\text { Schweizerdichtung, Volk } \\
\text { und Staat seit zwei Jahr- } \\
\text { hunderten (für Hörer aller } \\
\text { Fakultäten) }\end{array}$ & Prof. Faesi & Ebd. & 22 \\
\hline & 430. & $\begin{array}{l}\text { Die kulturellen Grundla- } \\
\text { gen des Kantons Zürich } \\
\text { (für Hörer aller Fakultäten) }\end{array}$ & $\begin{array}{l}\text { Kantonsschulprof. } \\
\text { Hunzinger }\end{array}$ & $\begin{array}{l}\text { Geschichte und ihre } \\
\text { Hilfswissenschaften }\end{array}$ & 25 \\
\hline \multirow[t]{2}{*}{ SS 1940} & 402. & $\begin{array}{l}\text { Lektüre und Interpretation } \\
\text { ausgewählter Abschnitte } \\
\text { aus Nietzsches „Wille zur } \\
\text { Macht““ }\end{array}$ & P.-D. E. Staiger & $\begin{array}{l}\text { Germanische Sprachen } \\
\text { und Literaturen }\end{array}$ & 22 \\
\hline & 483. & $\begin{array}{l}\text { Volkslied und Volkslied- } \\
\text { forschung (mit besond. } \\
\text { Berücksichtigung der } \\
\text { Schweiz) }\end{array}$ & $\begin{array}{l}\text { P.-D. Prof. Cherbu- } \\
\text { liez }\end{array}$ & $\begin{array}{l}\text { Kunst- und Musikwissen- } \\
\text { schaft }\end{array}$ & 26 \\
\hline \multirow[t]{6}{*}{$\begin{array}{l}\text { WS } \\
1940 / 41\end{array}$} & 382. & $\begin{array}{l}\text { Lektüre schweizerischer } \\
\text { Schauspiele der Reforma- } \\
\text { tionszeit }\end{array}$ & P.-D. Prof. Gröger & $\begin{array}{l}\text { Germanische Sprachen } \\
\text { und Literaturen }\end{array}$ & 22 \\
\hline & 389. & $\begin{array}{l}\text { Dramatiker des 19. Jahr- } \\
\text { hundts. }\end{array}$ & Prof. Ermatinger & Ebd. & 22 \\
\hline & 392. & $\begin{array}{l}\text { Theorie und Geschichte } \\
\text { der deutschen Tragödie }\end{array}$ & P.-D. E. Staiger & Ebd. & 22 \\
\hline & 393. & $\begin{array}{l}\text { Kolloquium über das Pro- } \\
\text { blem des Tragischen und } \\
\text { der tragischen Kunst }\end{array}$ & Ders. & Ebd. & 22 \\
\hline & 296. & Analyse von Dramen & Prof. Ermatinger & Deutsches Proseminar & 22 \\
\hline & 447. & $\begin{array}{l}\text { Die kulturellen Grundla- } \\
\text { gen des Kantons Zürich } \\
\text { (für Hörer aller Fakultäten) }\end{array}$ & $\begin{array}{l}\text { Kantonsschulprof. } \\
\text { Hunzinger }\end{array}$ & $\begin{array}{l}\text { Geschichte und ihre } \\
\text { Hilfswissenschaften }\end{array}$ & 25 \\
\hline SS 1941 & 414. & $\begin{array}{l}\text { Das Drama des 19. Jahr- } \\
\text { hunderts }\end{array}$ & Prof. Ermatinger & Deutsches Seminar & 22 \\
\hline
\end{tabular}


Theaterwissenschaftliche Vorlesungen der Universität Zürich, 1933-1945 (Fortsetzung)

\begin{tabular}{|c|c|c|c|c|c|}
\hline Semester & Nr. & Titel der Veranstaltung & Dozent & Fachbereich & S. \\
\hline \multirow[t]{4}{*}{$\begin{array}{l}\text { WS } \\
1941 / 42\end{array}$} & 398. & $\begin{array}{l}\text { Lessing, Herder und der } \\
\text { Sturm und Drang }\end{array}$ & Prof. Ermatinger & $\begin{array}{l}\text { Germanische Sprachen } \\
\text { und Literaturen }\end{array}$ & 22 \\
\hline & 400. & Schillers Leben und Werk & Prof. Ermatinger & Ebd. & 22 \\
\hline & 401. & $\begin{array}{l}\text { G. Hauptmann und der } \\
\text { Naturalismus }\end{array}$ & Prof. Faesi & Ebd. & 22 \\
\hline & 405. & $\begin{array}{l}\text { Lektüre von M. Heideg- } \\
\text { gers „Hölderlin und das } \\
\text { Wesen der Dichtung“ }\end{array}$ & P.-D. E. Staiger & Ebd. & 22 \\
\hline \multirow[t]{3}{*}{ SS 1942} & 408. & $\begin{array}{l}\text { Komik, Witz und Humor; } \\
\text { Kolloquium }\end{array}$ & P.-D. E. Staiger & Ebd. & 22 \\
\hline & 412. & Analyse von Dramen & Prof. Ermatinger & Deutsches Proseminar & 22 \\
\hline & 415. & Hebbels Dramen & Ders. & Deutsches Seminar & 22 \\
\hline \multirow[t]{7}{*}{$\begin{array}{l}\text { WS } \\
1942 / 43\end{array}$} & 405. & Goethe & Ders. & $\begin{array}{l}\text { Germanische Sprachen } \\
\text { und Literaturen }\end{array}$ & 22 \\
\hline & 406. & $\begin{array}{l}\text { Der Briefwechsel zwi- } \\
\text { schen Goethe und Schiller } \\
\text { und die klassische Ästhe- } \\
\text { tik; Kolloquium }\end{array}$ & P.-D. E. Staiger & Ebd. & 22 \\
\hline & 408. & $\begin{array}{l}\text { Dramatiker des 19. Jahr- } \\
\text { hundts. }\end{array}$ & Prof. Ermatinger & Ebd. & 22 \\
\hline & 411. & $\begin{array}{l}\text { *Dichtung und Geistesle- } \\
\text { ben der deutschen } \\
\text { Schweiz }\end{array}$ & Ders. & Ebd. & 22 \\
\hline & 468. & $\begin{array}{l}\text { Die kulturellen Grundla- } \\
\text { gen des Kantons Zürich } \\
\text { (für Hörer aller Fakultäten) }\end{array}$ & $\begin{array}{l}\text { Kantonsschulprof. } \\
\text { Hunzinger }\end{array}$ & Ebd. & 25 \\
\hline & 481. & $\begin{array}{l}\text { Das Theater der Griechen } \\
\text { und Römer, Bau und Spiel }\end{array}$ & Prof. v. Salis & $\begin{array}{l}\text { Kunst- und Musikwissen- } \\
\text { schaft }\end{array}$ & 25 \\
\hline & 501. & Wagners Musikdramen & P.-D. Prof. F. Gysi & $\begin{array}{l}\text { Kunstgeschichtliches Se- } \\
\text { minar }\end{array}$ & 25 \\
\hline \multirow[t]{2}{*}{ SS 1943} & 434. & $\begin{array}{l}\text { Hebbel, Ibsen und neuere } \\
\text { Dramatiker }\end{array}$ & Prof. Faesi & $\begin{array}{l}\text { Germanische Sprachen } \\
\text { und Literaturen }\end{array}$ & 23 \\
\hline & 438. & $\begin{array}{l}\text { Tendenzen der Literatur- } \\
\text { wissenschaft: Bespre- } \\
\text { chungen von germanisti- }\end{array}$ & P.-D. M. Wehrli & Ebd. & 24 \\
\hline
\end{tabular}


Theaterwissenschaftliche Vorlesungen der Universität Zürich, 1933-1945 (Fortsetzung)

\begin{tabular}{|c|c|c|c|c|c|}
\hline Semester & Nr. & Titel der Veranstaltung & Dozent & Fachbereich & S. \\
\hline & & $\begin{array}{l}\text { schen Neuerscheinungen } \\
\text { der letzten Jahre }\end{array}$ & & & \\
\hline \multirow[t]{2}{*}{$\begin{array}{l}\text { WS } \\
1943 / 44\end{array}$} & 414. & $\begin{array}{l}\text { Tendenzen der Literatur- } \\
\text { wissenschaft: Bespre- } \\
\text { chungen von germanisti- } \\
\text { schen Neuerscheinungen } \\
\text { der letzten Jahre }\end{array}$ & P.-D. M. Wehrli & Ebd. & 23 \\
\hline & 415. & $\begin{array}{l}\text { Die Elemente des Thea- } \\
\text { ters, mit Übungen }\end{array}$ & Dr. Eberle & Ebd. & 23 \\
\hline \multirow[t]{5}{*}{ SS 1944} & 425. & Das deutsche Lustspiel & Prof. E. Staiger & Ebd. & 23 \\
\hline & 429. & $\begin{array}{l}\text { Theaterwissenschaftliche } \\
\text { Übungen }\end{array}$ & $\begin{array}{l}\text { Prof. Faesi, Dr. } \\
\text { Georgette Boner }\end{array}$ & Ebd. & 23 \\
\hline & 434. & Schillers Ästhetik & Prof. E. Staiger & Deutsches Seminar & 24 \\
\hline & 484. & $\begin{array}{l}\text { Sitte und Brauch im Leben } \\
\text { des Volkes }\end{array}$ & P.-D. R. Weiß & $\begin{array}{l}\text { Geschichte und ihre } \\
\text { Hilfswissenschaften }\end{array}$ & 26 \\
\hline & 513. & Die Technik der Oper & Prof. Faesi & $\begin{array}{l}\text { Musikwissenschaftliches } \\
\text { Seminar }\end{array}$ & 28 \\
\hline \multirow[t]{6}{*}{$\begin{array}{l}\text { WS } \\
1944 / 45\end{array}$} & 415. & $\begin{array}{l}\text { Das deutsche Drama von } \\
\text { Kleist bis Grillparzer }\end{array}$ & Prof. E. Staiger & $\begin{array}{l}\text { Germanische Sprachen } \\
\text { und Literaturen }\end{array}$ & 23 \\
\hline & 416. & $\begin{array}{l}\text { Kolloquium (nur für Vor- } \\
\text { gerückte): Interpretation } \\
\text { von Dramen }\end{array}$ & Ders. & Ebd. & 23 \\
\hline & 419. & Dramaturgische Übungen & Dr. Wälterlin & Ebd. & 23 \\
\hline & 471. & $\begin{array}{l}\text { Sinn und Aufgabe vater- } \\
\text { ländischer Geschichte (für } \\
\text { Hörer aller Fakultäten) }\end{array}$ & Prof. v. Muralt & $\begin{array}{l}\text { Geschichte und ihre } \\
\text { Hilfswissenschaften }\end{array}$ & 26 \\
\hline & 472. & $\begin{array}{l}\text { Die kulturellen Grundla- } \\
\text { gen des Kantons Zürich } \\
\text { (für Hörer aller Fakultäten) }\end{array}$ & $\begin{array}{l}\text { Kantonsschulprof. } \\
\text { Hunzinger }\end{array}$ & Ebd. & 26 \\
\hline & 478. & $\begin{array}{l}\text { Die volkstümliche Kultur } \\
\text { des schweizerischen Al- } \\
\text { pengebiets }\end{array}$ & P.-D. Rich. Weiß & Ebd. & 26 \\
\hline SS 1945 & 433. & $\begin{array}{l}\text { Gerhart Hauptmann und } \\
\text { der Naturalismus }\end{array}$ & Prof. Faesi & $\begin{array}{l}\text { Germanische Sprachen } \\
\text { und Literaturen }\end{array}$ & 23 \\
\hline
\end{tabular}


Theaterwissenschaftliche Vorlesungen der Universität Zürich, 1933-1945 (Fortsetzung)

\begin{tabular}{|c|c|c|c|c|c|}
\hline Semester & Nr. & Titel der Veranstaltung & Dozent & Fachbereich & S. \\
\hline \multirow[t]{5}{*}{$\begin{array}{l}\text { WS } \\
1945 / 46\end{array}$} & 425. & $\begin{array}{l}\text { Die Anfänge des deut- } \\
\text { schen Dramas (13. bis } \\
\text { 16. Jahrhundert) }\end{array}$ & P.-D. M. Wehrli & Ebd. & 23 \\
\hline & 427. & Goethe & Prof. E. Staiger & Ebd. & 24 \\
\hline & 429. & $\begin{array}{l}\text { Hebbel, Ibsen (für Hörer } \\
\text { aller Fakultäten) }\end{array}$ & Prof. Faesi & Ebd. & 24 \\
\hline & 485. & $\begin{array}{l}\text { Die kulturellen Grundla- } \\
\text { gen des Kantons Zürich } \\
\text { (für Hörer aller Fakultäten) }\end{array}$ & $\begin{array}{l}\text { Kantonsschulprof. } \\
\text { Hunzinger }\end{array}$ & $\begin{array}{l}\text { Geschichte und ihre } \\
\text { Hilfswissenschaften }\end{array}$ & 27 \\
\hline & 501. & $\begin{array}{l}\text { Das antike Theater (mit } \\
\text { Kolloquium) }\end{array}$ & Prof. Fiechter & Kunstwissenschaft & 28 \\
\hline
\end{tabular}

